Revue d'histoire de l'Amérique française

ARS REVUE D.HISTOIRE DE L'AMÉRIQUE FRANÇAISE

\title{
Index des titres d'article ou de note de recherche
}

Index, 1987

Index des volumes XXXI-XL (juin 1977 - printemps 1987)

URI : https://id.erudit.org/iderudit/305683ar

DOI : https://doi.org/10.7202/305683ar

Aller au sommaire du numéro

Éditeur(s)

Institut d'histoire de l'Amérique française

ISSN

0035-2357 (imprimé)

1492-1383 (numérique)

Découvrir la revue

Citer ce document

(1987). Index des titres d'article ou de note de recherche. Revue d'histoire de l'Amérique française, 49-56. https://doi.org/10.7202/305683ar d'utilisation que vous pouvez consulter en ligne.

https://apropos.erudit.org/fr/usagers/politique-dutilisation/ 


\section{Index des titres d'article ou de note de recherche}


(NR) indique une note de recherche.

À la recherche de la nation: Maurice Séguin (1918-1984), 38, 4: 569-590 (Jean-Pierre WALLOT)

Agriculture de la Mauricie et du Québec 18501950, L', 35, 4: 537-562 (Normand SÉGUIN)

Allemands en Louisiane de 1721-1732, Les, 33, 1: 51-65 (Reinhart KONDERT)

Alliances matrimoniales exogames dans le Gouvernement de Québec 1700-1760, Les, 35, 1: 3-32 (Céline CYR, Guy DINEL, Jacques MATHIEU, Jeannine POZO et Jacques ST-PIERRE)

Alphabétisation de la population de la ville de Québec de 1750 à 1849, L', 39, 1: 51-76 (Michel-VERRETTE)

Aménagement rural et maîtrise de l'espace au $X X e$ siècle par les collectivités paysannes des régions défavorisées en France et au Québec (NR), 35, 2: 253-261 (FrançoiseEugénie PETIT)

Amérique du Nord et la Sacrée Congrégation "de Propaganda Fide", 1622-1799 - Guides et inventaires, L', 33, 2: 197-214 (Luca CODIGNOLA)

Annaotaha et Dollard vus de l'autre côté de la palissade, 35, 2: 163-178 (John A. DICKINSON)

Aperçu sur le commerce et le crédit à Québec 1820-1830, 36, 4: 527-551 (George BERVIN)

Appropriation foncière et bourgeoisie marchande: éléments pour une analyse de l'économie marchande du Bas-Canada avant 1846, 36, 2: 163-194 (Gérald BERNIER et Daniel SALÉE)

Archambault, Le Père Joseph-Papin, s. j. et l'École sociale populaire - Témoignage -, 35, 4: 563-587 (Richard ARÈS)

Art militaire et technique de guerre: le Fort de Chambly de 1710-1711, 37, 1: 21-49 (Marc LAFRANCE)

Artisans du cuir à Québec (1660-1760), Les, 34, 3: 341-356 (Marîse THIVIERGE)

Attitudes devant la mort d'après les clauses testamentaires dans le Gouvernement de Québec sous le Régime français, Les, 32 , 1: 57-94 (Marie-Aimée CLICHE)

Attitudes envers la langue française pendant les années 1840 (NR), 34, 2: 257-267 (Stephen KENNY)
Bibllothèque scientlfique de l'abbé Léon Provancher, La, 34, 4: 535-556 (Raymond DUCHESNE)

Blanchet, François, et le mouvement réformiste en médecine au début du XIXe siècle, 34, 2: 223-244 (Jacques BERNIER)

“Capricieuse" au Canada, La, 31, 3: 351-370 (Jacques PORTES)

Carrière politique de René Chaloult: l'art de promouvoir une politique nationaliste tout en sauvegardant son avenir politique, La, 39, 1: 25-50 (Denis CHOUINARD et Richard JONES)

Chapitre dans l'histoire de l'éducation des adultes au Québec: les écoles du soir, 1889-1892, Un, 34, 4: 597-625 (Ruby HEAP)

Charpentier, Alfred, face au gouvernement du Québec, 1935-1946, 31, 2: 211-227 (Denys CHOUINARD)

Classes dominantes au Québec, 1760-1840. Bilan historiographique, Les, $38,2: 223$ 243 (Fernand OUELLET)

Clergé nicolétain, 1885-1904: aspects sociographiques , Le (NR), 35, 3: 383-395 (Jean ROY)

Colonisation, climat et âge au baptême des Canadiens au XVIle siècle, 38, 3: 341-356 (Hubert CHARBONNEAU)

Colonisation de la seigneurie de Batiscan aux $17 e$ et 18 e siècles: l'espace et les hommes, La, 40, 2: 163-191 (Philippe JARNOUX)

Colonisation et rapatriement au Lac-SaintJean (1895-1905), 38, 3: 379-408 (Robert G. LEBLANC)

Colonisation française de l'Illinois: une réévaluation, La (NR), 39, 4: 583-591 (Margaret Kimball BROWN)

Comment se forme un ministre colonial. L'initiation de Colbert 1651-1664, 37, 3: 431452 (Philip P. BOUCHER)

Commerce, crédit et transactions foncières: pratiques de la communauté marchande du bourg de l'Assomption, 1748-1791, 39 , 3: 323-343 (Lise ST-GEORGES)

Commerce de détail au Canada (1870-1880), Le, 33, 4: 521-556 (Gaétan GERVAIS)

Commerce du blé et la navigation dans le Bas-Richelieu avant 1849, Le, 38, 1: 5-21 (P.-André SÉVIGNY)

Commerce triangulaire entre les ports terreneuviers français, les pêcheries d'Amé- 
rique du Nord et Marseille au 18e siècle: nouvelles perspectives, Le, 40, 2: 193-214 (Jean-François BRIÈRE)

Commis des trésoriers généraux de la marine à Québec - Nicolas Lanoullier de Boisclerc, Un, 32, 2: 181-202 (John KEYES)

Commissariat canadien à Paris (1882-1928), Le, 34, 3: 357-376 (Bernard PÉNISSON)

Comportement démographique des marchands de Montréal vers 1760, Le, 33, 3: 427-445 (José E. IGARTUA)

Conception de la littérature chez JosephSabin Raymond, 32, 4: 585-602 (Guy PROVOST)

Conceptions prénuptiales dans la vallée du Saint-Laurent avant 1725, Les (NR), 40, 2: 253-272 (Réal BATES)

Confréries dans le gouvernement de Québec sous le Régime français, Les, 39, 4: 491522 (Marie-Aimée CLICHE)

Congrès des métiers et du travail et la guerre civile espagnole, Le (NR), 33, 4: 575-581 (Richard MARTIN)

Conquête de 1760 et le problème de la transition au capitalisme, La, 39, 3: 369-389 (Claude COUTURE)

Conseil législatif et les bureaux d'enregistrement (1836), Le, 35, 2: 217-230 (Evelyn KOLISH)

Construction domiciliaire à Québec 1810 1820, La, 31, 4: 547-561 (Jacques BERNIER)

Construction navale à Québec, 1760-1825: sources inexplorées et nouvelles perspectives de recherches, La, 35, 2: 231-251 (Pierre DUFOUR)

Contestataires pragmatiques: les JeuneCanada, 1932-1938, Des, 40, 1: 5-28 (Denis CHOUINARD)

Coutume de Paris et la transmission des terres - Le rang de la Beauce à CalixaLavallée de 1730 à 1975, La, 34, 3: 331-339 (Pauline DESJARDINS)

Début des affaires publiques à la télévision québécoise 1952-1957, Le, 36, 2: 213-239 (Gérard LAURENCE)

Démographie différentielle en NouvelleFrance: villes et campagnes, 38, 3: 357 378 (Hubert CHARBONNEAU, Lorraine GADOURY et Yves LANDRY)

Députés conservateurs-nationalistes et le gouvernement Borden - le cas d'Albert
Sévigny (1911-1913), Les, 35, 1: 33-54

(Réal BÉLANGER)

Dernier effort de la France au Canada secours ou fraude? -, Le, 36, 3: 413-426 (Gilles PROULX)

Développement des disparités régionales en Gaspésle 1760-1960, Le, 36, 4: 483-506 (Maryse GRANDBOIS)

Diversification de l'activité économique de Timothy Hibbard Dunn, commerçant de bois à Québec, 1850-1898, La, 35, 3: 323336 (John KEYES)

Dix dernières années de la Revue d'histoire de l'Amérique française 1972-1981, Les (NR), 36, 4: 553-567 (Jean-Paul COUPAL)

Du Québec vers les Etats-Unis: l'étude des lieux d'origine (NR), 38, 3: 409-422 (Jean LAMARRE et Bruno RAMIREZ)

Dynamique communautaire et l'évolution des sociétés rurales québécoises aux $19 \mathrm{e}$ et 20e siècles. Construction d'un modèle, La, 40, 1: 51-71 (Gérard BOUCHARD)

Éditoriaux de La Presse, 1934-1936: une défense de la démocratie libérale, Les (NR), 33, 3: 451-462 (Yves FRENETTE)

Éducation et classes sociales: analyse de l'idéologie conservatrice-cléricale et petite bourgeoise - au Québec au milieu du 19e siècle, 32, 2: 159-179 (Nadia F.-EID)

Effet du déséquilibre des sexes sur le comportement matrimonial: comparaison entre la Nouvelle-France, Saint-Domingue et la Nouvelle-Orléans, L', 39, 2: 211-231 (Paul LACHANCE)

Égalitarisme paysan dans l'ancienne société rurale de la vallée du Saint-Laurent: éléments pour une ré-interprétation, L', 40, 3: 373-407 (Christian DESSUREAULT)

Élection partielle du quartier-ouest de Montréal en 1832: analyse politico-sociale, L', 32, 4: 565-584 (France GALARNEAU)

Émergence d'une scène politique: mouvement ouvrier et mouvements de réforme urbaine à Montréal au tournant du siècle Essai d'interprétation -, L' , 37, 2: 185-199 (Annick GERMAIN)

Endettement de l'évêché de Saint-Hyacinthe au XIXe siècle: le rôle décisif de Charles LaRocque dans l'extinction de cette dette, L', 33, 4: 557-574 (Réal BOUCHER)

Enfants abandonnés à Montréal au 19e siècle: la Crèche d'Youville des Soeurs Grises, 
1820-1871, Les, 40, 4: 537-559 (Peter GOSSAGE)

Engagements pour la traite des fourrures évaluation de la documentation, Les, 34 , 1: 3-26 (Gratien ALLAIRE)

Engagés pour le Canada partis de Nantes (1725-1732), Les (NR), 33, 4: 583-586 (Gabriel DEBIEN)

Entreprises de pêche à la morue de Joseph Cadet 1751-1758, Les, 37, 4: 557-572 (Mario MIMEAULT)

Espace, territoire et culture en NouvelleFrance: une vision géographique, 37,3 : 417-429 (Serge COURVILLE)

État, entrepreneurs, habitants et monopole: le "privilège" de la pêche au marsouin dans le Bas Saint-Laurent 1700-1730, 37, 4: 543555 (Alain LABERGE)

État et le commerce de la morue de TerreNeuve en France au XVIlle siècle, L', 36, 3: 323-338 (Jean-François BRIÈRE)

Étude de mentalité: les injures verbales au Canada au XVIlle siècle (1712-1748), Une (NR), 31, 2: 229-238 (André LACHANCE)

Exemple de peinture d'histoire au Québec: Charles Huot à l'Assemblée nationale, Un (NR), 31, 3: 397-405 (Raymond MONTPETIT)

Facteurs d'apparition du syndicalisme catholique dans l'imprimerie et les déterminants de la stratégie syndicale 1921-1945, Les, 37, 2: 241-269 (Luc DESROCHERS)

Faire l'histoire de la littérature française du XIXe siècle québécois, 38, 4: 523-547 (Manon BRUNET)

Famille, parenté et colonisation en NouvelleFrance (NR), 39, 3: 391-405 (Yves BEAUREGARD, Serge GOUDREAU, Andrée HÉROUX, Michèle JEAN, Alain LABERGE, Rénald LESSARD, Johanne NOEL et Lucie PAQUET)

Femme d'atfaires en Nouvelle-France: MarieAnne Barbel, veuve Fornel, Une, 31, 2: 165-185 (Lilianne PLAMONDON)

Femmes dans l'oeuvre de Groulx, Les, 32, 3: 385-398 (Susan Mann TROFIMENKOFF)

Financement et industrie en Mauricie, 19001950, 40, 1: 29-50 (Claude BELLAVANCE, Normand BROUILLETTE et Pierre LANTHIER)

Formation matérielle de la classe ouvrière à Montréal entre 1790 et 1830, La, 33, 1: 3950 (Robert TREMBLAY)
Garderies au XIXe siècle: les salles d'asile des Soeurs Grises à Montréal, Des, 34, 1 : 27-55 (Micheline DUMONT-JOHNSON)

Gestion des seigneuries de Gabriel Chrlstie dans la vallée du Richelieu (1760-1845), La, 40, 4: 561-582 (Françoise NOEL)

Gougou de Champlain et les croyances algonquiennes, Le (NR), 35, 3: 377-381 (Norman CLERMONT)

Grands thèmes de l'histoire de la famille aux États-Unis, Les, 39, 2: 185-209 (Tamara K. HAREVEN)

Grève de Lachute (1947), La, 37, 2: 271-289 (Denyse BAILLARGEON)

Grève des charretiers à Montréal, 1864, La, 31, 3: 371-395 (Margaret HEAP)

Grève des ouvriers de la construction navale à Québec (1840), La, 37, 2: 227-239 (Robert TREMBLAY)

Groulx et l'histoire: interrogation sur le passé en vue d'une direction d'avenir, 32, 3: 347356 (Pierre TOUSIGNANT)

Groulx éveilleur de conscience nationale (Concours Lionel-Groulx), 32, 3: 435-448 (Christiane HUOT)

Groulx, historien d'hier et d'aujourd'hui, Le Chanoine (Concours Lionel-Groulx), 32, 3: 459-464 (Jean SIROIS)

Groulx historiographe, 32, 3: 407-433 (JeanPierre WALLOT)

Groulx, Lionel: bibliographie (1964-1979), 32, 3: 465-523 (Juliette RÉMILLARD)

Groulx, Lionel, cet inconnu?, 32, 3: 325-346 (Benoît LACROIX)

Groulx, Lionel, historien d'hier ou d'aujourd'hui (Concours Lionel-Groulx), 32, 3: 455-458 (Suzanne GAGNÉ)

Guerre iroquoise et la mortalité en NouvelleFrance 1608-1666, La, 36, 1: 31-54 (John A. DICKINSON)

Histoire et la recherche du sens. Discours de réception à l'Académie canadienne-française, L', 37, 4: 533-542 (Jean-Pierre WALLOT)

Historiographie des sciences et des techniques au Canada, 35, 2: 193-215 (Raymond DUCHESNE)

Hommage à Lionel Groulx, 32, 3: 323 (Jacques MATHIEU)

Hôpital et société en Nouvelle-France: l'HôtelDieu de Québec à la fin du XVIle siècle, 31 , 1: 29-47 (François ROUSSEAU) 
Implantation et expansion de l'Union internationale des travailleurs en chaussures au Québec de 1900 à 1940, 36, 1: 75-105 (Jacques ROUILLARD)

Industrie de l'amiante au Québec, 1878-1929, L', 33, 2: 187-195 (Robert ARMSTRONG) Industrie de la chaussure à Montréal: 18401870 - Le passage de l'artisanat à la fabrique, L', 31 , 2: 187-210 (Joanne BURGESS)

Industries et politiques minières au Québec: une analyse économique 1896-1975, 37, 4: 573-602 (Pierre PAQUETTE)

Influence de l'école des Annales au Québec, L', 33, 3: 357-386 (Alfred DUBUC)

Insurrection appréhendée et l'administration de la justice au Canada: le point de vue d'un historien, L', 34, 1: 57-93 (F. Murray GREENWOOD)

Intelligentsia du Québec et la migration des Canadiens français vers l'Ouest canadien, 1870-1930, L', 33, 2: 163-185 (A.-N. LALONDE)

Intervention à Rome dans la lutte pour le suffrage féminin au Québec (1922), Une, 32, 1: 3-18 (Luigi TRIFIRO)

Introduction à l'étude de la société saguenayenne auX XIXe et XXe siècles, 31, 1: 327 (Gérard BOUCHARD)

Introduction de la faillite au Bas-Canada: conflit social ou national?, L', 40, 2: 215-235 (Evelyn KOLISH)

Inuit du Labrador méridional face à l'exploitation canadienne et française des pêcheries (1700-1760), Les, 31, 4: 481-499 (François TRUDEL)

Inventaire descriptif des documents de la session, 1841-1936, Un (NR), 31, 4: 563-574 (Christian POUYEZ)

Investisseurs français et le nationalisme canadien-français. La Compagnie de colonisation et de crédit des Cantons de l'Est 1881-1893, Les, 32, 1: 19-39 (Jack I.

\section{LITTLE)}

Liberté de presse à la fin du XIXe siècle: le cas de Canada-Revue, La, 31, 4: 501-523 (Jean DE BONVILLE)

Ligue de l'Enseignement (1902-1904): héritage du passé et nouveaux défis, La, 36 , 3 : 339-373 (Ruby HEAP)

Lutte pour le droit à l'avortement (1969-1981), La, 37, 1, 81-90: (Diane LAMOUREUX)

Maladies de la misère: aspects de la santé publique à Montréal 1880-1914, Les, 36, 4: 507-526 (Martin TÉTREAULT)
Manuel de l'abbé Thomas Maguire et la langue québécolse au XIXe siècle, Le, 35 , 3: 337-354 (André LAPIERRE)

Marchand rural en Nouvelle-France - François-Augustin Bailly de Messein, 17091771, Un, 33, 2: 215-262 (Louis MICHEL)

Mariage et cadre de vie familiale dans une paroisse ouvrière montréalaise: SainteBrigide, 1900-1914, 39, 2: 233-251 (Lucia FERRETTI)

Mères ou malades: les Québécoises de l'entre-deux-guerres vues par les médecins, 38, 1: 23-37 (Andrée LÉVESQUE)

Mesure de l'influence du chanoine Lionel Groulx sur son milieu, 32, 3: 357-384 (François-Albert ANGERS)

Milieu d'origine du clergé québécois 17751840: mythes et réalités, Le, 37, 3: 373-397 (Serge GAGNON et Louise LEBELGAGNON)

Militantisme des travailleurs au Québec et en Ontario, niveau de syndicalisation et mouvement de grève (1900-1980), Le, 37, 2 : 201-225 (Jacques ROUILLARD)

Montpetit, Édouard, et l'université moderne, ou l'échec d'une génération, 36, 1: 3-29 (Marcel FOURNIER)

Mouvements sociaux, la violence et les interventions armées au Québec 1867-1967, 37, 1: 67-79 (Jean PARISEAU)

Mutations de la Confédération des travailleurs catholiques du Canada (1940-1960), 34, 3 : 377-405 (Jacques ROUILLARD)

Naissance et déclin d'une élite locale: la Banque des Cantons de l'Est, 1859-1912, 38, 2: 165-179 (Ronald RUDIN)

Naissances illegitimes sur les rives du SaintLaurent avant 1730, Les (NR), 40, 2: 239252 (Réal BATES et Lyne PAQUETTE)

Nicollet dans le lac Supérieur et non dans le lac Michigan, Jean, 34, 2: 183-196 (Marcel TRUDEL)

Notables du Saguenay au 20 e siècle à travers deux corpus blographiques, Les, $39,1: 3$ 23 (Gérard BOUCHARD, France MARKOWSKI et Yves OTIS)

Obligation scolaire et travail des enfants au Québec: 1900-1950, 38, 1: 39-58 (Thérèse HAMEL)

Oeuvre de Guy Frégault, L', 35, 1: 55-68 (Jean-Charles FALARDEAU)

Officiers et marchands: les sociétés de commerce des fourrures, 1715-1760, 40, 3: 409-428 (Gratien ALLAIRE) 
On a vieilli Champlain, 32, 2: 229-237 (Jean LIEBEL)

Ordre et désordre: le Montreal Lunatic Asylum et la naissance de l'asile au Québec, 39, 3: 345-367 (André CELLARD et Dominique NADON)

Origine acadienne de Moncton: le Coude, L', 37, 3: 399-416 (Raoul DIONNE)

Origines des travailleurs de l'Alcan au Saguenay, 1925-1939, Les, 37, 2: 291-308 (José E. IGARTUA et Marine DE FRÉMINVILLE)

Parents québécois et l'État canadien au début du programme des allocations familiales: 1944-1955, Les, 40, 1: 73-95 (Dominique JEAN)

Paris devant l'opinion canadienne-française: les récits de voyages entre 1820 et 1914, 38, 4: 549-568 (Serge JAUMAIN)

Patriote inconnu: Joseph-Édouard Turcotte, Un (NR), 38, 1: 59-71 (Louisette POTHIER)

Patriotes et le gouvernement responsable dans les années 1830, Les, 33, 1: 25-37 (F. Murray GREENWOOD)

Patronat et entreprise au XXe siècle: I'exemple mauricien, 38, 2: 181-201 (Claude BELLAVANCE)

«Persécution et exil»: la venue au Québec des congrégations françaises, 1900-1914, 36, 3: 389-411 (Guy LAPERRIÈRE)

Pour redécouvrir notre 16e siècle: les pêches à Terre-Neuve d'après les archives notariales de Bordeaux, 39, 4: 523-549 (Laurier TURGEON)

Pour une méso-histoire du XIXe siècle canadien, 33, 3: 387-425 (Gilles PAQUET et Jean-Pierre WALLOT)

Premiers journaux du district de Saint-François (1823-1845), Les (NR), 31, 2: 239-253 (Jean-Pierre KESTEMAN)

Présence protestante en Nouvelle-France, La, 31, 3: 325-349 (Marc-André BÉDARD)

Projet d'histoire sociale de la population du Saguenay: I'appareil méthodologique, Le, 32, 1: 41-56 (Gérard BOUCHARD et Yolande LAVOIE)

Projet de musée national à Québec à l'époque du peintre Joseph Légaré (1833-1853), Un (NR), 31, 1: 75-82 (John R. PORTER)

Projet Riel, Le (NR), 33, 3:, 447-450: (George F. G. STANLEY)

Propagande de l'École sociale populaire en faveur du syndicalisme catholique 1911 .
1949, La, 32, 2: 203-228 (Jean-Claude STAMANT)

Québec et le monde communiste: Cowansville 1931, Le, 34, 2: 171-182 (Andrée LÉVESQUE)

Quelques aspects du niveau de richesse et de la vie matérielle des artisans de Québec et de Montréal, 1740-1755, 40, 3: 339372 (Jean-Pierre HARDY)

Quelques comportements des Canadiens au XVIle siècle d'après les registres paroissiaux, 31, 1: 49-73 (Hubert CHARBONNEAU, Yves LANDRY et Raymond ROY)

Question des écoles du Manitoba - Un nouvel éclairage, La, 33, 1: 3-23 (Gilbert-L. COMEAULT)

Questions internationales dans les premiers inédits de Lionel Groulx (1895-1909), Les, 34, 2: 245-255 (Réjean BERGERON et Yves DROLET)

Qui perd sa langue, perd sa foi? (NR), 33, 2: 263-265 (Jean-Olivier FERRON et François VAILLANCOURT)

Rameau de Saint-Père et le métier d'historien, 33, 3: 331-355 (Lise TRÉPANIER et Pierre TRÉPANIER)

Ramezay et sa famille, au Canada et en France, après 1760, Jean-Baptiste-Nicolas Roch de (NR), 37, 4: 603-610 (Raymonde LITALIEN)

Rapport agriculture - forêt au Québec, note historiographique (NR), Le, 33, 1: 67-78 (Guy GAUDREAU)

Réception de La Lanterne par la presse canadienne-française (NR), 34, 2: 269-274 (Francis PARMENTIER)

Récit de M. de Courcelles au lac Ontario (1671), et Dollier de Casson, Le (NR), 32, 2: 239-250 (Jean MARMIER)

Récits de voyages de Pierre-Esprit Radisson Étude d'histoire bibliographique, Les (NR), 34, 3: 407-414 (Pierre GERMAIN)

Registre de population de la Nouvelle-France: un outil pratique au service de la démographie historique et de l'histoire sociale, Le (NR), 38, 3: 423-426 (Yves LANDRY)

Régulation sociale et répression de la déviance au Bas-Canada au tournant du 19e siècle (1791-1815), 38, 4: 499-521 (Jean-Marie FECTEAU)

Rente du faubourg Saint-Roch A Québec 1750-1850, La, 34, 4: 569-596 (Louise DECHÊNE) 
Représentativité de l'inventaire après décès I'étude d'un cas: Québec au début du XIXe siècle, La, 34, 4: 515-533 (Yvan MORIN)

Réseau d'enseignement public bas-canadien, 1841-1867: une institution de l'État libéral, Le, 40, 4: 505-535 (Jean-Pierre CHARLAND)

Rôle de Lionel Groulx comme éveilleur de conscience nationale de ses compatriotes (Concours Lionel-Groulx), Le, 32, 3: 449454 (Joanne BOURASSA)

Rôle du blé à Montréal sous le Régime seigneurial, Le, 36, 2: 241-262 (Corinne BEUTLER)

Rome et le Canada: la mission de Mgr Conroy, 33, 4: 499-519 (Nive VOISINE)

Roy, Camille, et Lionel Groulx: la querelle de L'Appel de la race, 32, 3: 399-405 (Lucie ROBERT)

Santé publique et organisation de la profession médicale au Québec 1870-1918, 35, 3: 355-375 (Claudine PIERRE-DESCHÊNES)

Société littéraire et historique de Québec (The Literary and Historical Society of Quebec) 1824-1890, La, 35, 2: 179-192 (Ginette BERNATCHEZ)

Soldat à Québec, 1748-1759, 32, 4: 535-563 (Gilles PROULX)

Sources archivistiques: leur utilisation dans l'étude de la bourgeoisie marchande bascanadienne (1800-1830), Les, 38, 2: 203222 (George BERVIN)

Sous les feux croisés de l'histoire et de l'anthropologie: la famille en Europe, 39, 2: 163-184 (Martine SÉGALEN)

Standardisation des études médicales et la consolidation de la profession dans la deuxième moitié du XIXe siècle, La, 37, 1: 51-65 (Jacques BERNIER)

Stratégie foncière de l'habitant: Québec (1790-1835), 39, 4: 551-581 (Gilles PAQUET et Jean-Pierre WALLOT)
Stratégie industrielle et développement régional: le cas de la Mauricie au XXe siècle, 37, 1: 3-19 (Pierre LANTHIER)

Sur quelques discontinuités dans l'expérience socio-économique du Québec: une hypothèse, 35, 4: 483-521 (Gilles PAQUET et Jean-Pierre WALLOT)

Sur quelques effets de la rupture structurelle engendrée par la Conquête au Québec: 1760-1854, 35, 1: 69-95 (Gérald BERNIER)

Syndicat catholique des allumettières de Hull, 1919-1924, Le, 32, 4: 603- 628 (Michelle LAPOINTE)

Système colonial des peuples modernes: un inédit de Rameau de Saint-Père, Du, 36, 1: 55-74 (Pierre TRÉPANIER)

Théories de la famille et rapports famille-pouvoirs dans le secteur éducatif au Québec et en France (1850-1960), 34, 2: 197-221 (Nadia FAHMY-EID et Nicole LAURINFRENETTE)

Thèse messianique et les Franco-Américains, La, 34, 4: 557-567 (André SÉNÉCAL)

Thompson, E.P., dans des arpents de neige: les historiens canadiens-anglais et la classe ouvrière, 37, 2: 165-184 (Desmond MORTON)

Travailleurs à la construction du chemin de fer dans la région de Sherbrooke (18511853), Les, 31, 4: 525-545 (Jean-Pierre KESTEMAN)

Tremblement de terre de 1663: les convulsions du verbe ou la mystification du logos chez Charlevoix, Le, 36, 3: 375-387 (Pierre BERTHIAUME)

Urbanisation et population: le cas de Montréal en 1861, 35, 4: 523-535 (Jean-Claude ROBERT)

Varlet, Dominique-Marie - de l'Église de Québec à la réforme d'Utrecht -, 36, 2: 195-212 (Serge A. THÉRIAULT) 